\title{
Unconventional phase transitions in strongly anisotropic 2D (pseudo)spin systems
}

\author{
Vitaly Konev ${ }^{1, \star}$, Evgeny Vasinovich ${ }^{1}$, Vasily Ulitko ${ }^{1}$, Yury Panov ${ }^{1}$, and Alexander Moskvin ${ }^{1}$ \\ ${ }^{1}$ Ural Federal University, Ekaterinburg, 620083, Russia
}

\begin{abstract}
We have applied a generalized mean-field approach and quantum Monte-Carlo technique for the model 2D $S=1$ (pseudo)spin system to find the ground state phase with its evolution under application of the (pseudo)magnetic field. The comparison of the two methods allows us to clearly demonstrate the role of quantum effects. Special attention is given to the role played by an effective single-ion anisotropy ("on-site correlation").
\end{abstract}

\section{Introduction}

These days spin algebra and spin Hamiltonians are used not only in the traditional fields of spin magnetism and magnetic resonance but in so-called pseudospin lattice systems with the on-site occupation constraint (see, e.g., Ref. [1]). Standard pseudospin formalism represents a variant of the equivalent operators technique widely known in different physical problems from classical and quantum lattice gases, binary alloys, (anti)ferroelectrics,.. to neural networks, however, usually for simplest $s=1 / 2$ pseudospin value. At variance with quantum $s=1 / 2$ systems the Hamiltonian of $S=1$ spin lattices in general is characterized by several additional terms such as a single ion anisotropy, biquadratic isotropic and anisotropic exchange couplings, that results in their rich phase diagrams and novel types of the order such as quantum paramagnet and spin-nematic order.

Typical $S=1$ spin Hamiltonian with an uniaxial onsite and exchange anisotropies reads as follows:

$$
\begin{aligned}
\hat{H}=\sum_{i>j} J_{i j}\left(S_{i x} S_{j x}+S_{i y} S_{j y}\right. & \left.+\lambda S_{i z} S_{j z}\right)+ \\
& +\sum_{i} D S_{i z}^{2}-\sum_{i} h S_{i z}
\end{aligned}
$$

The spectrum of the spin Hamiltonian (1) in the absence of external magnetic field changes drastically as $D$ varies from very small to very large positive or negative values. A strong "easy-plane" anisotropy for large positive $D>0$ favors a singlet phase where spins are in the $S_{z}=0$ ground state. This "quadrupole" phase has no magnetic order, and is aptly referred to as a quantum paramagnetic phase (QPM), which is separated from the "ordered" state by a quantum critical point (QCP) at some $D=D_{c}$. This is a quadrupole state with no magnetic order, so that all linear order parameters vanish and

\footnotetext{
^e-mail: konefvitbka53@gmail.com
}

only a quadrupole (spin-nematic) order parameter such as $Q_{z z}=\left\langle S_{z}^{2}-\frac{2}{3}\right\rangle$ is nonzero. The QPM phase consists of a unique ground state with total spin $S_{z}^{\text {total }}=0$, separated by a gap from the first excited states, which lie in the sectors $S_{z}^{\text {total }}= \pm 1$. This is purely a quantum phase without a counterpart in the classical models. The QPM order differs in principle from the conventional paramagnetic state, because for $S=1$ in the classical paramagnetic state $\left\langle S_{x}^{2}\right\rangle=\left\langle S_{y}^{2}\right\rangle=\left\langle S_{z}^{2}\right\rangle=2 / 3$, while in the quantum paramagnetic state $\left\langle S_{z}^{2}\right\rangle=0,\left\langle S_{x}^{2}\right\rangle=\left\langle S_{y}^{2}\right\rangle=1$.

Quite a few compounds can be mentioned with the quantum paramagnetic ground state of the considered type which are described by the Hamiltonian (1), these are several $\mathrm{Ni}^{2+}$ compounds with a real $S=1$ ground state and $\mathrm{Fe}^{2+}$ compounds $\mathrm{CsFeBr}_{3}, \mathrm{CsFeCl}_{3}$ with a ground state described by the fictitious $\mathrm{S}=1$ spin (pseudospin) due to strong spin orbit coupling (see, e.g., Ref. [2] and references therein).

However, studying anisotropic $S=1$ spin and pseudospin systems is of a great importance not only for quantum magnets but for different bosonic-like systems with the on-site Hilbert space truncated to the three lowest occupation states $n=0,1,2$, in particular, for so-called semi-hard core bosons. Furthermore, the $S=1$ pseudospin formalism was suggested to describe the charge degree of freedom in a model high- $\mathrm{T}_{c}$ cuprate with the on-site Hilbert space reduced to the only triplet of the three effective valence centers $\left[\mathrm{CuO}_{4}\right]^{7-, 6-, 5-}$ (nominally $\mathrm{Cu}^{1+; 2+; 3+}$ ), where the electronic and lattice degrees of freedom get strongly locked together [3-6]. Indeed, recently [3] it was argued that an unique property of high- $\mathrm{T}_{c}$ cuprates is related with a dual nature of the Mott insulating state of the parent compounds that manifests itself in two distinct energy scales for the charge transfer $(\mathrm{CT})$ reaction: $\mathrm{Cu}^{2+}+\mathrm{Cu}^{2+} \rightarrow \mathrm{Cu}^{1+}+\mathrm{Cu}^{3+}$. The $d-d$ CT gap as derived from the optical measurements in parent cuprates such as $\mathrm{La}_{2} \mathrm{CuO}_{4}$ is $1.5-2.0 \mathrm{eV}$ while the true (thermal) $d-d \mathrm{CT}$ 
gap, or effective correlation parameter $U_{d}$, appears to be as small as $0.4-0.5 \mathrm{eV}$. It means the cuprates should be addressed to be $d-d$ CT unstable systems whose description implies accounting of the three many-electron valence states, or nominally the $\mathrm{Cu}^{1+, 2+, 3+}$ centers, on an equal footing as a well-defined charge triplet. Effective $S=1$ pseudospin Hamiltonian for such a cuprate does incorporate all the on-site and inter-site couplings, three types of correlated one-particle (fermionic) transport and a twoparticle (bosonic) charge transfer with a charge density constraint. With the exception of the three-spin terms the model becomes equivalent to a generalized strongly anisotropic $S=1$ quantum magnet in an external magnetic field with a constraint on the magnetization.

The Hamiltonian implies possible simplifications, in particular, so-called negative- $U(U=2 D)$ model, which in the limit of large negative $U$ becomes equivalent to the model of hard-core bosons. For the model we made use of a special algorithm for CUDA architecture for NVIDIA graphics cards, a nonlinear conjugate-gradient method to minimize energy functional, and Monte-Carlo technique for large square lattices $256 \times 256$ to directly observe the forming of the ground state configuration with lowering the temperature and its evolution under deviation from half-filling [7]. The technique allowed us to examine earlier implications and uncover novel features of the phase transitions, in particular, look upon the nucleation of different metastable topological structures, in particular, odd domain structure with emergence of filamentary superconductivity nucleated at the antiphase domain walls in the insulating charge-ordered phase.

Here, in the paper, we have applied a generalized mean-field approach and quantum Monte-Carlo (QMC) technique for a simplified model 2D $S=1$ (pseudo)spin system to find the ground state phase with its evolution under application of the (pseudo)magnetic field. Despite the generalized MFA implies a quantum-mechanical treatment of the $S=1$ sites in a molecular field the more comprehensive QMC technique allowed us to clearly uncover the role of quantum effects.

\section{$2 S=1$ (pseudo)spin Hamiltonian}

The $S=1$ spin algebra includes the eight nontrivial independent spin operators: spin-dipole moment $\mathbf{S}$ and five spin-quadrupole operators $Q_{i j}=\left(\frac{1}{2}\left\{S_{i}, S_{j}\right\}-\frac{2}{3} \delta_{i j}\right)$ whose mean values define so-called spin-nematic order. Spin operators $S_{ \pm}$and $T_{ \pm}=\left\{S_{z}, S_{ \pm}\right\}$change the pseudospin projection (and occupation number!) by \pm 1 , while $S_{ \pm}^{2}$ changes the pseudospin projection by \pm 2 .

Hereafter in the paper we will focus on a simplified 2D $S=1$ (pseudo)spin Hamiltonian for bosonic-like systems with the on-site Hilbert space truncated to the three lowest occupation states $\mathrm{n}=0,1,2$, in particular, for so-called semi-hard core bosons, or for model high- $\mathrm{T}_{c}$ cuprate with the on-site Hilbert space reduced to the only triplet of the three effective valence centers $\left[\mathrm{CuO}_{4}\right]^{7-, 6-, 5-}$ (nominally $\left.\mathrm{Cu}^{1+; 2+; 3+}\right)$. We restrict ourselves by the nearest neighbour coupling and the only two-particle transport term (inter- site biquadratic anisotropy) so the (pseudo)spin Hamiltonian takes the form as follows:

$$
\begin{aligned}
\hat{H}= & \sum_{i}\left(\Delta S_{i z}^{2}-\mu S_{i z}\right)+V \sum_{\langle i j\rangle} S_{i z} S_{j z}- \\
& -t \sum_{\langle i j\rangle}\left(S_{i+}^{2} S_{j-}^{2}+S_{i-}^{2} S_{j+}^{2}\right),
\end{aligned}
$$

where $V>0, t>0$. The first single-site term in $\hat{H}$ describes the effects of a bare pseudo-spin splitting and relates with the on-site density-density interactions, or correlations: $\Delta=U / 2$. The second term, or a pseudospin Zeeman coupling may be related with a pseudo-magnetic field $\| z$ which acts as a chemical potential $\mu$ for boson systems with a boson density constraint:

$$
\frac{1}{N} \sum_{i}\left\langle S_{i z}\right\rangle=n
$$

where $n$ is the deviation from a half-filling $(n=0)$.

The third (Ising) term in $\hat{H}$ describes the effects of the short- and long-range inter-site density-density interactions. The last term in $\hat{H}$ describes the two-particle intersite hopping. In the strong on-site attraction limit of the model (large easy-axis pseudospin on-site anisotropy) we arrive at the Hamiltonian of the hard-core, or local, bosons which was earlier considered to be a starting point for explanation of the cuprate high- $\mathrm{T}_{c}$ superconductivity [8]. The spin counterpart of $\hat{H}$ corresponds to an anisotropic $S=1$ magnet with a single ion (on-site) and two-ion (bilinear and biquadratic) symmetric anisotropy in an external magnetic field. It describes an interplay of the Zeeman, single-ion and two-ion anisotropic terms giving rise to a competition of an (anti)ferromagnetic order along $Z$-axis with an in-plane $X Y$ spin-nematic order. A remarkable feature of the Hamiltonian (2) is that the on-site pseudospin states $M=0$ and $|M|=1$ do not mix under the inter-site coupling. The model allows us to directly study a continuous transformation of the semi-hard-core bosons to the effective hard-core bosons formed by boson pairs under driving the correlation parameters $\Delta=U / 2$ to large negative values ("negative- $U$ model"). The simplified model can be directly applied to a description of bosonic systems with suppressed one-particle hopping.

\section{Mean-field approximation}

To analyse the simplified model we start with a mean-field approximation (MFA) for 2D square lattice, however, at variance with a conventional classical MFA we made use of more correct approach that takes into account the quantum nature of the $S=1$ (pseudo)spin states [9]. First we introduce a set of the on-site $S=1$ coherent states

$$
|\mathbf{c}\rangle=c_{-1}|-1\rangle+c_{0}|0\rangle+c_{+1}|+1\rangle,
$$

where the $c_{M}$ coefficients can be represented as follows

$$
c_{1}=\sin \frac{\theta}{2} \cos \frac{\phi}{2} e^{-i \frac{\alpha}{2}}, c_{0}=\cos \frac{\theta}{2} e^{i \frac{\beta}{2}}, c_{-1}=\sin \frac{\theta}{2} \sin \frac{\phi}{2} e^{i \frac{\alpha}{2}}
$$



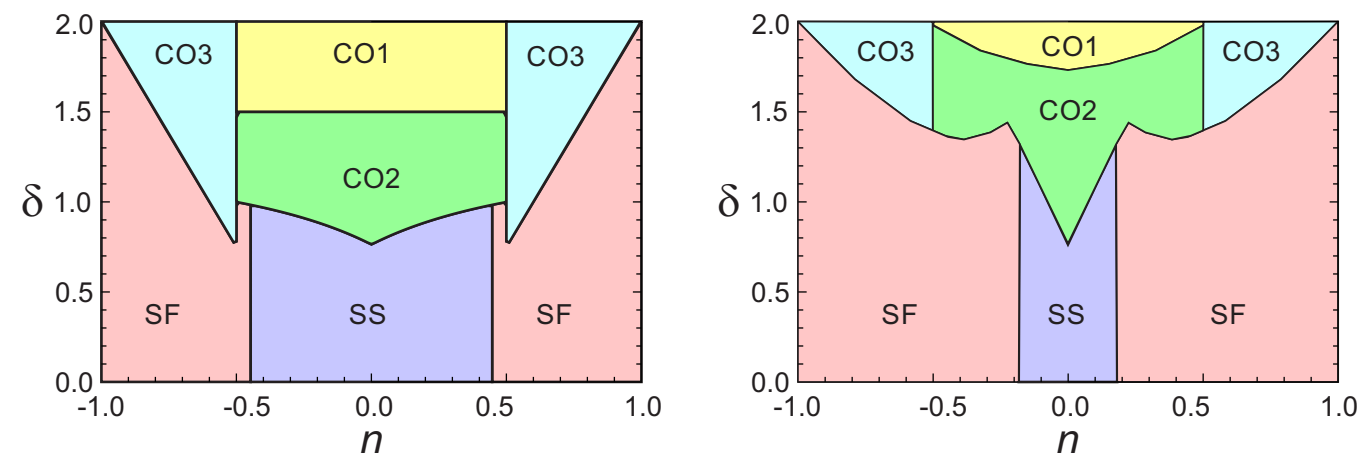

Figure 1. (Color online) The $\delta$ - $n$ ground state phase diagrams for the model system given $V / t=0.75$ (left panel, the MFA results, right panel, the QMC results).

with $\theta, \phi, \alpha, \beta$ to be parameters defined by the minimization of the energy. The MFA energy can be written as follows

$$
\begin{gathered}
E=\frac{\Delta}{2} \sum_{i}\left(1-\cos \theta_{i}\right)-\frac{\mu}{2} \sum_{i}\left(1-\cos \theta_{i}\right) \cos \phi_{i}+ \\
+\frac{V}{4} \sum_{\langle i j\rangle}\left(1-\cos \theta_{i}\right)\left(1-\cos \theta_{j}\right) \cos \phi_{i} \cos \phi_{j}- \\
-\frac{t}{8} \sum_{\langle i j\rangle}\left(1-\cos \theta_{i}\right)\left(1-\cos \theta_{j}\right) \sin \phi_{i} \sin \phi_{j} \cos \left(\alpha_{i}-\alpha_{j}\right) .
\end{gathered}
$$

It is worth noting that the energy does not depend on $\beta$. Below we denote $\delta=\Delta / t$ and $v=V / t$. In a two-sublattice A-B model which seems to be a reasonable approximation for the system with the nearest-neighbor coupling we arrive at a high-temperature non-ordered (NO) phase and the five MFA uniform phases, two phases with nonzero local superfluid order parameter, or pseudospin nematic order $\left\langle S_{A, B \pm}^{2}\right\rangle \neq 0$ and three charge ordered phases with $\left\langle S_{A, B \pm}^{2}\right\rangle=0$ but different types of the sublattice occupation (pseudospin $S_{z}$ components):

Superfluid (SF) phase: $\left\langle S_{A, B z}\right\rangle=n,\left\langle S_{A, B z}^{2}\right\rangle=1$, $\left\langle S_{A, B \pm}^{2}\right\rangle=\frac{\zeta}{2} \sqrt{1-n^{2}} e^{ \pm i \alpha}$, uncertain factor $\zeta= \pm 1$.

Supersolid (SS) phase: $\left\langle S_{A, B z}^{2}\right\rangle=1$,

$$
\left\langle S_{A, B z}\right\rangle=n \mp \sqrt{1+n^{2}-\frac{4|n| v}{\sqrt{4 v^{2}-1}}},
$$

$$
\begin{aligned}
& \left\langle S_{A, B \pm}^{2}\right\rangle=\frac{\zeta}{2} e^{ \pm i \alpha} \times \\
& \times\left(\sqrt{|n| \sqrt{\frac{2 v+1}{2 v-1}}-n^{2}} \pm \operatorname{sgn} n \sqrt{|n| \sqrt{\frac{2 v-1}{2 v+1}}-n^{2}}\right) .
\end{aligned}
$$

Charge ordered CO1 phase: $\left\langle S_{A z}\right\rangle=0,\left\langle S_{A z}^{2}\right\rangle=0$, $\left\langle S_{B z}\right\rangle=2 n,\left\langle S_{B z}^{2}\right\rangle=2|n|,(|n| \leq 0.5)$.

Charge ordered CO2 phase: $\left\langle S_{A z}\right\rangle=2 n-\operatorname{sgn} n,\left\langle S_{A z}^{2}\right\rangle=$ $1-2|n|,\left\langle S_{B z}\right\rangle=\operatorname{sgn} n,\left\langle S_{B z}^{2}\right\rangle=1,(|n| \leq 0.5)$.

Charge ordered CO3 phase: $\left\langle S_{A z}\right\rangle=\operatorname{sgn} n,\left\langle S_{A z}^{2}\right\rangle=1$, $\left\langle S_{B z}\right\rangle=2 n-\operatorname{sgn} n,\left\langle S_{B z}^{2}\right\rangle=2|n|-1,(|n| \geq 0.5)$.
Table 1. The energies of the MFA ground-state phases.

\begin{tabular}{ll}
\hline Phase & $E /(t N)$ \\
\hline $\mathrm{SF}$ & $\delta-1+n^{2}(2 v+1)$ \\
$\mathrm{SS}$ & $\delta-2 v+2|n| \sqrt{4 v^{2}-1}$ \\
$\mathrm{CO} 1$ & $|n| \delta$ \\
$\mathrm{CO} 2$ & $(1-|n|) \delta+4\left(|n|-\frac{1}{2}\right) v$ \\
$\mathrm{CO} 3$ & $|n| \delta+4\left(|n|-\frac{1}{2}\right) v$ \\
\hline
\end{tabular}

The energies of the MFA ground state phases are listed in Table 1. Note that the energy of $\mathrm{CO} 1$ phase does not depend on inter-site interaction parameter $v$.

The density of superfluid component is related to helicity modulus [10]. This allows us to find an expression for the superfluid density $\rho$ in terms of the local superfluid order parameters in the two-sublattice MFA:

$$
\rho=\operatorname{Re}\left(\left\langle S_{A+}^{2}\right\rangle\left\langle S_{B-}^{2}\right\rangle\right) .
$$

The superfluid density in the SF phase, $\rho=\left(1-n^{2}\right) / 4$, has a maximum value at $n=0$ and does not depend on the the inter-site density-density interaction parameter $v$. In the SS phase, the superfluid density $\rho=|n| /\left(2 \sqrt{4 v^{2}-1}\right)$ decreases with rising of $v$.

Interestingly, all the local order parameters do not depend on the correlation parameter $\Delta$, while this parameter governs the energy of different phases. Taking into account the on-site correlations we arrive at very rich and intricate phase diagrams for the model system as compared with relatively simple phase diagrams for hard-core bosons $[8,11]$. In Fig. 1 (left panel) we present an example of the MFA $\delta$ - $n$ phase diagrams calculated given $v=0.75$. At half-filling $n=0$ the positive values of the correlation parameter $\delta$ stabilize a limiting $\mathrm{CO} 1$ phase with $\left\langle S_{A, B z}\right\rangle=$ $\left\langle S_{A, B z}^{2}\right\rangle=0$, or a "parent $\mathrm{Cu}^{2+}$ phase" for a model cuprate, while positive values of $v$ stabilize a limiting $\mathrm{CO} 2$ phase with $\left\langle S_{A, B z}\right\rangle= \pm 1 ;\left\langle S_{A, B z}^{2}\right\rangle=1$, or a checkerboard "antiferromagnetic" order of pseudospins along $z$-axis, or a disproportionated $\mathrm{Cu}^{1+}-\mathrm{Cu}^{3+}$ phase for a model cuprate. As a result of the competition between the on-site and intersite correlations we arrive at a "starting" CO1 phase for 

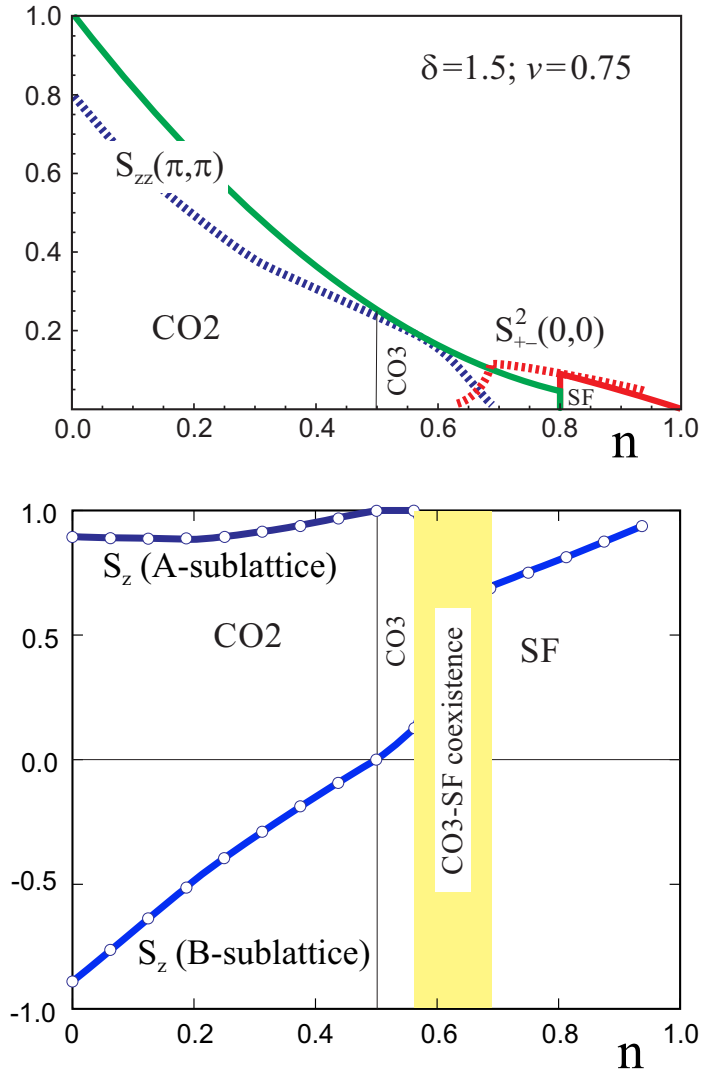

Figure 2. (Color online) Top panel: Correlation functions for the model $S=1$ pseudospin system given $\Delta / t=1.5, V / t=0.75$, solid lines are the MFA results, dotted lines are the QMC results. Bottom panel: QMC data for the sublattice $S_{z}$-components as functions of the deviation from the half-filling. Filling points to a $\mathrm{CO} 3-\mathrm{SF}$ coexistence phase typical for the first kind phase transition.

$\delta>2 v$ or $\mathrm{CO} 2$ phase for $\delta \leq 2 v$. At $n=0.5$ we see a transformation of the $\mathrm{CO} 1$ and $\mathrm{CO} 2$ phases into the $\mathrm{CO} 3$ phase. The line of the first order phase transition CO3-SF in Fig. 1 corresponds to the equality of the respective energies. It is worth to note that the critical concentration $n$ for the SS-SF, CO1, CO2-CO3 transitions does not depend on the correlation parameter $\delta$. In Fig. 2 (top panel, solid lines) we present the $n$-dependence of the correlation functions $S_{z z}(\pi, \pi)=\left\langle S_{z}, S_{z}\right\rangle$ (static structure factor) and $S_{+-}^{2}(0,0)=\left\langle S_{+}^{2}, S_{-}^{2}\right\rangle$ at $\delta=1.5, v=0.75$, determining the long-range CO and SF orders, respectively, given $\delta=1.5$, that is in an immediate closeness to $\mathrm{CO} 2-\mathrm{CO} 1$ phase transition for small $n$.

\section{Quantum Monte-Carlo calculations}

We have performed QMC calculations for our model Hamiltonian (2). In our QMC calculations, we use the D. Galanakis open source program [12]. This program is an implementation of the Stochastic Green Function algorithm for the simulation of bosonic Hamiltonians [13]. During the simulation, we performed $5 \times 10^{6}$ Monte-Carlo steps for equilibration and $10 \times 10^{6}$ Monte-Carlo steps for measurement the values under investigation in the square lattice $12 \times 12$ with periodical boundary conditions. In Fig. 1 (right panel) we compare the ground state $\delta$ - $n$ phase diagram of our model 2D system calculated on square lattice $12 \times 12$ given $v=0.75$ with that of calculated within a generalized MFA approach (left panel). First of all, it should be noted that the QMC calculations do not "obey" the MFA two-sublattice restrictions and at $n \neq 0$ all the QMC phases are inhomogeneous (disordered) phases confined by the concentration constraint. Nevertheless, the configuration averaging does lead to results similar those for the $\mathrm{CO} 1, \mathrm{CO} 2$, and $\mathrm{CO} 3$ phases of the two-sublattice MFA model.

As for simple hard-core counterpart [8, 11], despite some qualitative agreement, we see rather large quantitative difference between two phase diagrams in Fig. 1. In particular, it concerns a clearly larger volume of the quantum SF phase that might be related with a sizeable suppression of quantum fluctuations within MFA approach. In Fig. 2 (top panel, two dotted lines) we present the QMC calculated static structure factor $S_{z z}(\pi, \pi)$ and the superfluid (pseudospin nematic) correlation function $S_{+-}^{2}(0,0)$. It is worth to note a semiquantitative agreement with the MFA data. Smaller value of the quantum structure factor $S_{z z}(\pi, \pi)$ at $n=0$ is believed to be a result of the pseudospin reduction due to quantum fluctuations. Bottom panel in Fig. 2 shows the $n$-dependence of the mean sublattice $S_{z}$ values, $S_{A z}$ and $S_{B z}$, that clearly demonstrates the pseudospin quantum reduction effect within $\mathrm{CO} 2$ phase and specific features of the sublattice occupation, or "pseudomagnetization" under $\mathrm{CO} 2-\mathrm{CO} 3-\mathrm{SF}$ transformation.

\section{Conclusions}

A simplified 2D $S=1$ pseudospin Hamiltonian with a twoparticle transport term (pseudospin nematic coupling) was analyzed within a generalized MFA and QMC technique. We have obtained the ground-state phase diagrams and correlation functions given different values of the coupling parameters with a focus on the role of the on-site correlation effect (single-ion anisotropy). The comparison of the two methods allows us to uncover fundamental shortcomings of the MFA technique and clearly demonstrate the role of quantum effects.

The research was supported by the Government of the Russian Federation, Program 02.A03.21.0006 and by the Ministry of Education and Science of the Russian Federation, projects Nos. 2277 and 5719 .

\section{References}

[1] C.D. Batista, G. Ortiz, Adv. Phys. 53, 1 (2004)

[2] A.V. Sizanov and A.V. Syromyatnikov, Phys. Rev. B 84, 054445 (2011)

[3] A.S. Moskvin, Phys. Rev. B 84, 075116 (2011)

[4] A.S. Moskvin, J. Phys.:Condens. Matter. 25, 085601 (2013)

[5] A.S. Moskvin, JETP. 121, 477 (2015)

[6] A.S. Moskvin, J. Phys.: Conf. Ser. 592, 012076 (2015) 
[7] A.S. Moskvin, Yu.D. Panov, F.N. Rybakov, A.B. Borisov, J. Supercond. Nov. Magn. 30, 43 (2017).

[8] R. Micnas, J. Ranninger, S. Robaszkiewicz, Rev. Mod. Phys. 62, 113 (1990)

[9] N.A. Mikushina, A.S. Moskvin, Phys. Lett. A 302, 8 (2002)
[10] M.E. Fisher, M.N. Barber, D. Jasnow. Phys. Rev. A. 8, 1111-1124 (1973).

[11] G. Schmid, S. Todo, M. Troyer, A. Dorneich, Phys. Rev. Lett. 88, 167208 (2002)

[12] https://github.com/galanakis/QMCSGF

[13] V.G. Rousseau, Phys. Rev. E 78, 056707 (2008) 